\title{
Quasi-Elastic Light Scattering Study of Concentration Dependence of Diffusion and Internal Motion in Chain Polymers
}

\author{
Izumi NISHIO* and Akiyoshi WADA** \\ Department of Physics, Faculty of Science, University of Tokyo, \\ 7-3-1, Bunkyo-ku, Tokyo 113, Japan.
}

(Received September 7, 1979)

\begin{abstract}
Solutions of polystyrene $\left(M_{w}=1.0 \times 10^{7}\right)$ in 2-butanone (ethyl methyl ketone), in a concentration range $0.1 \mathrm{mg} \mathrm{cc}^{-1}$ to $10 \mathrm{mg} \mathrm{cc}^{-1}$, were studied using Quasi-Elastic Light Scattering. The relation of diffusion-like fluctuations in highly concentrated polymer solutions (cooperative diffusion) to the internal motion of single polymers was explored. We observed that when the concentration of a solution was brought from a dilute region to a highly concentrated region, the Rayleigh line spectrum clearly showed that the internal motion had changed from intramolecular motion within a single polymer to cooperative diffusion. We also analyzed the relaxation time of the lowest mode of the internal motion of single polymers in terms of the bead and spring model.

KEY WORDS Polystyrene/Quasi-Elastic Light Scattering/Intramolecular Motion / Cooperative Diffusion /
\end{abstract}

The Rayleigh line in the spectrum of light scattered from a dilute solution of random coil polymers provides informations about the translational diffusion and intramolecular motions of solute chain molecules. ${ }^{1}$ Since 1972, much work ${ }^{2,3}$ concerning the intramolecular motion of polystyrene has been carried out using the Quasi-Elastic Light Scattering (QELS) technique. By these experiments, the relaxation time of the lowest mode of the intramolecular motion was determined. The results of these experiments show fair agreement with the relaxation time calculated by the free-draining model with measured values of $D$ and $\left\langle l_{\mathrm{e}}{ }^{2}\right\rangle$, where $D$ is the diffusion coefficient of the polymer and $\left\langle l_{\mathrm{e}}^{2}\right\rangle$ is the mean square end-to-end distance of the polymer. These experiments were performed using very dilute solutions in which intermolecular interactions were negligible.

When a solution has a finite concentration, the Rayleigh spectrum is expected to be quite different from that of a dilute solution of the same polymers, due to the intermolecular interactions. Indeed, Geissler et $a l^{4}$ measured the spectrum of light

* Present Address: Rm 13-2033, Physics Department, M.I.T., Cambridge, MA 02139, U.S.A.

** To whom all correspondence should be addressed. scattered from a highly concentrated polystyrene solution and found the spectrum quite different from that of a dilute solution. They showed that the correlation function of intensity fluctuations in the scattered light from the highly concentrated polystyrene solution had the form of a single exponential, and that the relaxation time of the exponential was related not to the diffusion of the whole polymer but to the diffusion of density fluctuations in the gel. Recently, Munch et $a l^{5}$ measured $D$, the average diffusion coefficient in polystyrene solutions ranging from dilute to highly concentrated. They showed that $D$ becomes large as the concentration of the solution increases in spite of the increase of viscosity of the solution.

A chain polymer has many orders of intramolecular motion with translational diffusion being only the zeroth order motion. It is very natural to think that not only diffusion but also the other modes will change as the polymer concentration is increased. In this report, we have studied the relation between the spectrum of light scattered from polystyrene in 2-butanone and the internal motion of the polymers as the polymer concentration varied from dilute solutions in which the molecules could be thought of as independent, to higher concentrations in which the molecules overlapped. 


\section{MATERIAL AND METHOD}

Polystyrene, $M_{w}=1.0 \times 10^{7}$, was prepared for us by Prof. H. Nagasawa of Nagoya University. The solvent, 2-butanone, was chosen because of its high specific refractive index increment for solutions of polystyrene. 2-Butanone was reagent grade and was carefully distilled to remove any dust. The solutions were prepared at room temperature and mixed by placing the sample vials horizontally at the perimeter of a vertical wheel rotating 12 times per minute. The sample with the highest concentration, $10 \mathrm{mg} \mathrm{cc}^{-1}$, dissolved in about 24 hours. In some cases, a solution was prepared at its final concentration by placing a weighed amount of solute in a weighed quantity of solvent; others were prepared by dilution of a highly concentrated solution. Samples made to the same concentration by both methods gave the same results. All solutions were centrifuged for 2 hours at 10,000 rpm and then carefully removed to airtight sample cells. For all samples, the absence of dust and polymer aggregations was confirmed by measuring the angular dependence of the scattering intensity. All measurements were made at $25.0 \pm 0.1^{\circ} \mathrm{C}$ to allow comparison with the results of other workers.

The concentration at which molecules just begin to overlap is defined by

$$
c^{*}=0.74 \times M_{w} /\left[N_{\mathrm{A}} \times(4 / 3) \pi R_{\mathrm{g}}{ }^{3}\right]
$$

where $N_{\mathrm{A}}$ is Avogadro's number, the factor 0.74 is the close-packed arrangement factor, and $R_{\mathrm{g}}$ is the radius of gyration of the polymer. For the polystyrene used in this work, eq 1 gives $c^{*}=1.54$ $\mathrm{mg} \mathrm{cc}^{-1}$, where the value used for $R_{\mathrm{g}}$ was taken from

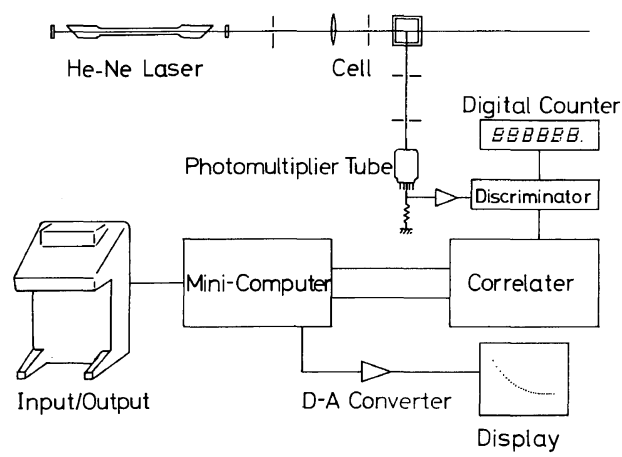

Figure 1. Blockdiagram of the Self-Beating Spectrometer. ref 2. We therefore chose concentrations ranging from $0.1 \mathrm{mg} \mathrm{cc}^{-1}$ (about $1 / 15$ of $c^{*}$ ) to $10 \mathrm{mg} \mathrm{cc}^{-1}$ (about 7 times $c^{*}$ ).

A self-beating spectrometer using single photon counting was used (see Figure 1). The incident beam, from an NEC GLG-108 He-Ne laser ( $50 \mathrm{~mW}$ ), was focused on the center of the sample cell. Two pinholes, separated by $15 \mathrm{~cm}$, were placed in the scattered beam. These pinholes determined the scattering angle and scattering volume. The scattered light was then detected on the photocathode of a Hamamatsu-TV R-374 photomultiplier tube. The photoelectron pulse from the load resister of the photomultiplier tube was amplified and converted to a TTL pulse using a discriminator with a variable threshold. The correlation function for the intensity fluctuations of the scattered light was calculated by a clipped correlater, UNION 301, having an HP$2100 \mathrm{~A}$ mini-computer as a data accumulator. The scattering intensity from the samples was continuously monitored by a digital counter.

The scattered electric field correlation function, $g^{(1)}(\tau)$, of a very dilute polymer solution is given by,

$$
\begin{aligned}
g^{(1)}(\tau) \propto\left\langle\left\{\sum_{l=1}^{N} \exp \left[i \boldsymbol{k}_{\mathrm{s}} \boldsymbol{r}_{l}(t+\tau)\right]\right\}\right. \\
\left.\times\left\{\sum_{m=1}^{N} \exp \left[-i \boldsymbol{k}_{\mathrm{s}} \boldsymbol{r}_{\mathrm{m}}(t)\right]\right\}\right\rangle
\end{aligned}
$$

where $N$ is the number of polymer segments, $\boldsymbol{r}_{l}(t)$ is the position of the $l$-th segment at time $t, \boldsymbol{k}_{\mathrm{s}}$ is the scattering vector, and $\langle>$ means time average. The correlation function for the intensity fluctuations in the scattering light, $g^{(2)}(\tau)$, is related to $g^{(1)}(\tau)$ by

$$
g^{(2)}(\tau)=\left|g^{(1)}(\tau)\right|^{2}
$$

Assuming that the motion of the center of the mass of the polymer is independent of the internal motion of the segments and obeys the diffusion equation, (eq 2) becomes,

$$
\begin{aligned}
& g^{(1)}(\tau) \propto \exp (\left.-D{k_{\mathrm{s}}}^{2} \tau\right) \times\left\langle\left\{\sum_{l=1}^{N} \exp \left[i \boldsymbol{k}_{\mathrm{s}} \boldsymbol{r}_{l}(t+\tau)\right]\right\}\right. \\
&\left.\times\left\{\sum_{m=1}^{N} \exp \left[-i \boldsymbol{k}_{\mathrm{s}} \boldsymbol{r}_{\mathrm{m}}{ }^{\prime}(t)\right]\right\}\right\rangle
\end{aligned}
$$

or,

$$
g^{(1)}(\tau)=\exp \left(-D k_{\mathrm{s}}{ }^{2} \tau\right) \times I(\tau)
$$

where $\boldsymbol{r}_{l}^{\prime}(t)$ is the position of the $l$-th segment relative 
to the center of mass of the polymer, and $I(\tau)$ is the contribution to the correlation function due to internal motion of the polymer.

Pecora ${ }^{1}$ calculated $g^{(1)}(\tau)$ using the bead and spring model of the chain polymer introduced by Zimm and Rouse. ${ }^{7-8}$ Pecora showed when $k_{\mathrm{s}}{ }^{2}$ is small enough to satisfy the relation,

$$
\chi=(1 / 6)\left\langle l_{\mathrm{e}}^{2}\right\rangle k_{\mathrm{s}}^{2}<1,
$$

$g^{(1)}(\tau)$ has a single exponential form:

$$
g^{(1)}(\tau) \infty \exp \left(-D k_{\mathrm{s}}{ }^{2} \tau\right) \text { or } I(\tau)=P_{0}(\chi)
$$

where $P_{0}(\chi)$ is the amplitude of the nonrelaxation part of $I(\tau)$, and $\chi$ is the dimensionless parameter introduced by Pecora. For the range of $k_{\mathrm{s}}^{2}$ that satisfies the condition $1<\chi<4$, Pecora ${ }^{1}$ showed that $g^{(1)}(\tau)$ has a double exponential form:

$$
\begin{aligned}
& g^{(1)}(\tau) \propto \exp \left(-D{k_{\mathrm{s}}}^{2} \tau\right) \\
& \quad \times\left\{P_{2}(\chi) \exp \left[-\left(2 / \tau_{1}\right) \tau\right]+P_{0}(\chi)\right\} \\
& =P_{0}(\chi) \exp \left(-D{k_{\mathrm{s}}}^{2} \tau\right) \\
& \quad+P_{2}(\chi) \exp \left\{-\left[D{k_{\mathrm{s}}}^{2}+\left(2 / \tau_{1}\right) \tau\right]\right\}
\end{aligned}
$$

or

$$
I(\tau)=P_{2}(\chi) \exp \left[-\left(2 / \tau_{1}\right) \tau\right]+P_{0}(\chi)
$$

where $\tau_{1}$ is the relaxation time of the lowest mode of the internal motion, and $P_{2}(\chi)$ is the amplitude of the relaxation part. The first term in eq 8 a corresponds to the diffusive motion while the second term corresponds to internal motion. When $\chi>5$, Pecora showed that $I(\tau)$ becomes multiexponential in form indicating the appearance of higher modes.

In this paper, the correlation function, $g^{(2)}(\tau)$, is analyzed by two methods. By the first method, we obtained 5 parameters, $A_{1}, A_{2}, T_{1}, T_{2}$, and $B$, by making a best fit of the correlation function, $c(\tau)$, to the double exponential formula,

$$
c(\tau)=\left[A_{1} \exp \left(-\tau / T_{1}\right)+A_{2} \exp \left(-\tau / T_{2}\right)\right]^{2}+B(9)
$$

where $T_{1} \geqq T_{2}$ is assumed. The constant term $B$ is introduced to compensate for the long term laser power fluctuations. If the relative base line amplitude, $B /\left(A_{1}+A_{2}\right)$, was greater than $0.5 \%$, the data was not used. Figure 2 shows some typical results of the 5 parameter fit. The accuracy of the fit, $A F$, is given by the inverse of the rms error of the fit normalized by $\left(A_{1}+A_{2}\right)^{2}$,
$A F=\left[(1 / n)\left\{\sum_{i=1}^{n}\left[g_{\text {obs. }}^{(2)}\left(t_{i}\right)-c\left(t_{i}\right)\right]^{2}\right\}^{1 / 2} /\left(A_{1}+A_{2}\right)^{2}\right]^{-1}$

where $n$ is the number of points used in the least squares fit; $g_{\mathrm{obs}}^{(2)}\left(t_{i}\right)$ is the observed correlation function at delay time $t_{i}$; and $c\left(t_{i}\right), A_{1}$, and $A_{2}$ are defined by eq 9 . Most of the data used in this work have values of $A F$ above 300. In the second method of analysis, the relaxation function associated with the intramolecular motion is obtained using the measured value of $D$ in the equation,

$$
I(\tau)=\left[g^{(2)}(\tau)\right]^{1 / 2} \exp \left(D k_{\mathrm{s}}{ }^{2} \tau\right) .
$$

This equation follows directly from eq 3 and 5.

\section{RESULTS}

\section{Dilute Solutions}

Figure 3 summarizes the results for the dilute solution, $0.1 \mathrm{mg} \mathrm{cc}^{-1}$, analyzed by the double exponential method. The parameter $\chi$ was calculated using the equation,

$$
\chi=(1 / 6)\left\langle l_{\mathrm{e}}^{2}\right\rangle k_{\mathrm{s}}^{2}
$$

where the value $\left\langle l_{\mathrm{e}}{ }^{2}\right\rangle=3.0( \pm 0.1) \times 10^{3} \AA$ was obtained from Figure 4. This value for $\left\langle l_{\mathrm{e}}^{2}\right\rangle^{1 / 2}$ is in good agreement with $\left\langle l_{\mathrm{e}}{ }^{2}\right\rangle^{1 / 2}=2980 \AA$, calculated from the experimental equation derived by McAdam et al. ${ }^{6}$ using $M_{w}=1.0 \times 10^{7}$.

As seen in Figure 3, the inverse of the slower relaxation time, $T_{1}^{-1}$, is linear in $\chi$ over the entire range of $\chi$ measured. This means that this slower relaxation time corresponds to $\left(D k_{\mathrm{s}}^{2}\right)^{-1}$. The measured value of $D, 6.2( \pm 0.1) \times 10^{-8} \mathrm{~cm}^{2} \mathrm{~s}^{-1}$, is in good agreement with the relation between $\log \langle D\rangle$ and $\log \left\langle M_{w}\right\rangle$ obtained by King et al. ${ }^{9}$ Using the Einstein-Stokes formula, the hydrodynamic radius is calculated as $8.8 \times 10^{-6} \mathrm{~cm}$. This is $72 \%$ of $R_{\mathrm{g}}$.

Within the range $1<\chi<4$, the plot of the inverse of the faster relaxation time, $T_{2}^{-1}$, is nearly parallel to that of the diffusion relaxation time, which agrees with $T_{2}^{-1}=D k_{\mathrm{s}}{ }^{2}+2 / \tau_{1}$ (see eq $8 \mathrm{a}$ ). In this case, we obtained $\tau_{1}^{-1}=2.0( \pm 0.2) \times 10^{3} \mathrm{~s}^{-1}$.

In the range where $\chi>4, T_{2}$ became much smaller, in accordance with the prediction of Pecora. ${ }^{1}$

Figure 5 shows an observed intramolecular relaxation curve, $I(\tau)$, reconstructed by the method given by eq 11 . In this figure, the solid line represents the curve calculated using the equation of Pecora $e t$ 


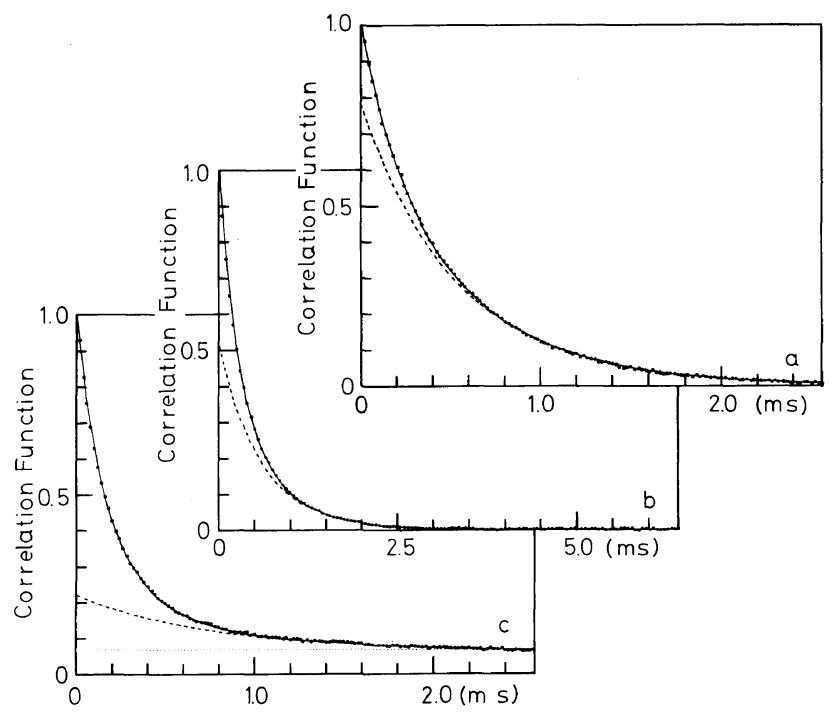

Figure 2. Observed correlation functions and their least-squares fits. The solid lines give the least-squares fit to a double exponential curve

$$
c(\tau)=\left[A_{1} \exp \left(-\tau / T_{1}\right)+A_{2} \exp \left(-\tau / T_{2}\right)\right]^{2}+B,
$$

the broken lines give $\left[A_{1} \exp \left(-\tau / T_{1}\right)\right]^{2}$, the pure translational part, and the dotted lines give $B$.

(a) Typical correlation function for a dilute sample at a small angle. Here, the concentration is $0.1 \mathrm{mg} \mathrm{cc}^{-1}$, and $k_{\mathrm{s}}^{2}=1.53 \times 10^{10} \mathrm{~cm}^{-2}$. Under this condition, the relaxation curve is considered to have a double exponential form as stated in the text. The results of the fit give,

$$
\begin{aligned}
& A_{1}=0.883 \pm 0.03, A_{2}=0.117 \pm 0.03 \\
& 1 / T_{1}=9.35 \pm 0.3 \times 10^{2} \mathrm{~s}^{-1} \\
& 1 / T_{2}=4.68 \pm 0.4 \times 10^{3} \mathrm{~s}^{-1} \\
& B=0.3 \times 10^{-2}, A F=397
\end{aligned}
$$

(b) Typical correlation function for a semi-dilute sample at a small angle. Here, the concentration is 3.0 mg cc${ }^{-1}$, and $k_{\mathrm{s}}{ }^{2}=1.39 \times 10^{10} \mathrm{~cm}^{-2}$. The intermolecular interaction appears as seen by the increase of the relative amplitude of the non-translational part of the correlation function compared to (a). Here the results of the fit give,

$$
\begin{aligned}
& A_{1}=0.714 \pm 0.02, A_{2}=0.286 \pm 0.02 \\
& 1 / T_{1}=8.39 \pm 0.3 \times 10^{2} \mathrm{~s}^{-1} \\
& 1 / T_{2}=3.13 \pm 0.3 \times 10^{3} \mathrm{~s}^{-1} \\
& B=0.11 \times 10^{-2}, A F=734
\end{aligned}
$$

(c) Typical correlation function for the highly concentrated sample at a small angle is shown. Here the concentration is $10 \mathrm{mg} \mathrm{cc}^{-1}$, and $k_{\mathrm{s}}{ }^{2}=1.53 \times 10^{10} \mathrm{~cm}^{-2}$. The results of the fit are,

$$
\begin{aligned}
& A_{1}=0.412 \pm 0.06, A_{2}=0.588 \pm 0.06 \\
& 1 / T_{1}=6.35 \pm 3.0 \times 10^{2} \mathrm{~s}^{-1} \\
& 1 / T_{2}=4.07 \pm 1.0 \times 10^{3} \mathrm{~s}^{-1} \\
& B=0.064, \quad A F=427 .
\end{aligned}
$$

Here, the rather high value of $B$ shows the existence of very slow relaxation, and that the double exponential analysis dose not work well, especially for determining the relaxation time.

$a l .^{1}$ The agreement between the curves is good for small values of $\chi$, but becomes poor when $\chi>5$. However when the relaxation time of the second mode is sufficiently slow compared to the first mode, there is better agreement (Figure 6). Here, although the theoretical curve has been calculated for $N=100$ segments, the values calculated for $P_{0}(\chi)$ and $P_{2}(\chi)$ in eq 8 a fit those calculated by Pecora for $N=\infty$ within 


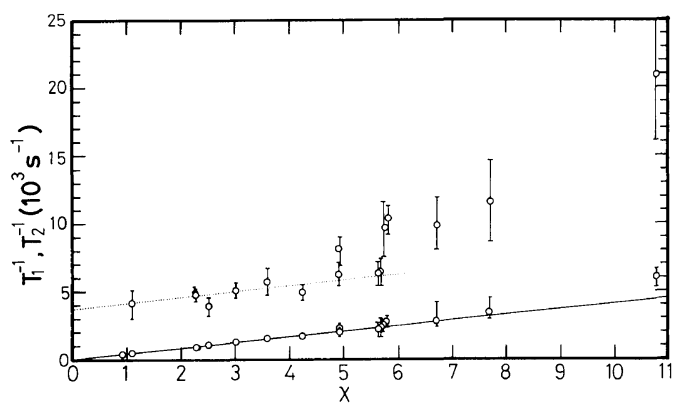

Figure 3. Results of the double exponential analysis for a dilute solution, $0.1 \mathrm{mg} \mathrm{cc}^{-1}$. The inverse of the larger relaxation time, $T_{1}{ }^{-1},(-\mathrm{O}-)$ and the inverse of the shorter relaxation time, $T_{2}{ }^{-1},(---\bigcirc---)$ are plotted against $\chi$. The solid line shows that $T_{1}{ }^{-1}$ fulfils Fick's law, $T_{1}^{-1} \propto D k_{\mathrm{s}}{ }^{2}$. The slope gives $D$, the translational diffusion coefficient of the polymer. Here, $D=6.2 \pm 0.1 \times 10^{-8}$ $\mathrm{cm}^{2} \mathrm{~s}^{-1}$. In the range $1<\chi<4$, the two curves are parallel. The intercept at $\chi=0$ of the dotted line with the vertical axis gives half the relaxation time of the lowest mode of the internal motion. Here, $\tau_{1}=0.52 \times 10^{-3} \mathrm{~s}$.

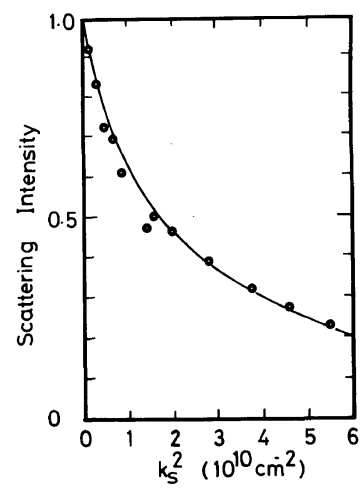

Figure 4. The scattering intensity of the $0.5 \mathrm{mg} \mathrm{cc}^{-1}$ solution is plotted against $k_{\mathrm{s}}{ }^{2}$. The solid line gives the theoretical scattering intensity of the polymer with $\left\langle l_{\mathrm{e}}^{2}\right\rangle^{1 / 2}=3.0 \times 10^{3} \AA$.

$2 \%$ accuracy. This shows $N=100$ is large enough for the range of $\chi, 0<\chi<10$, used in this work.

\section{Semi-dilute and Highly Concentrated Solutions}

Figure 7 shows the effects of polymer concentration on the shape of the correlation functions. The change in shape of the curves first appears in a correlation function corresponding to a high value of $k_{\mathrm{s}}^{2}$. This means that the first signs of change in the intermolecular interaction are

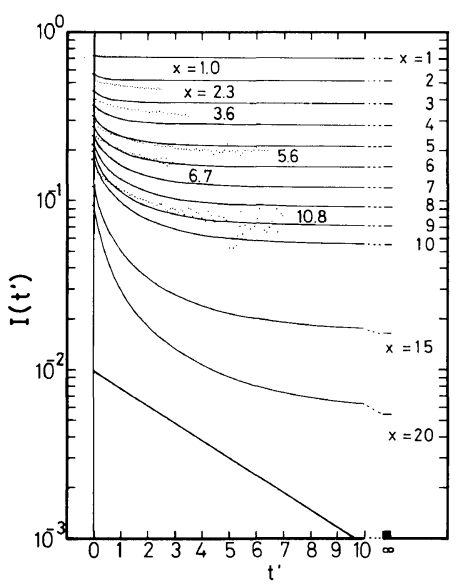

Figure 5. Relaxation curves of the internal motion for various $\chi$ values are shown. They were constructed by the second method from the values of $c(\tau)$ and $D$ observed in the $0.1 \mathrm{mg} \mathrm{cc}^{-1}$ solution and are plotted by dots. The initial value of each curve is chosen to give the relative scattering intensity assuming $\left\langle l_{\mathrm{e}}{ }^{2}\right\rangle^{1 / 2}=3.0 \times 10^{3} \AA$. The solid lines show the prediction of the free-draining model for $N=100$ at $\chi=1,2,3,4,5,6,7,8,9,10,15$, and 20 . The parameters, $b^{2}$ and $\beta$, of the free-draining model were fixed to give the same relaxation time, $\tau_{1}$, as our observations. Relaxation curves were plotted against the normalized time, $t^{\prime} \propto \tau / \chi$, for all values of $\chi$. Thus the diffusion part is always represented by the same curve, the straight line at the bottom of the figure.

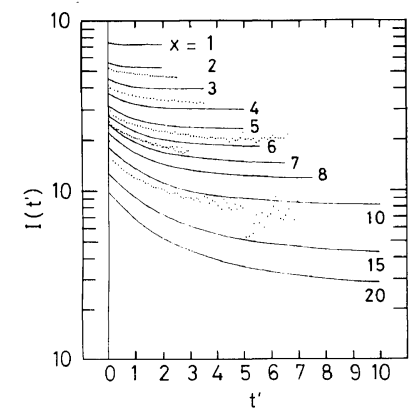

Figure 6. This is the same as Figure 5, except the theoretical curves were calculated under the assumption $\tau_{k}^{-1}=0$, for $k \geqq 2$, where $\tau_{k}$ is the relaxation time of the $k$ th mode of the internal motion.

associated with rather small dimensions or short decay periods. At increased concentrations, the change appears for even smaller values of $k_{\mathrm{s}}{ }^{2}$. The characteristics of these changes are (a) the appearance of a long tail on the correlation function, and (b) the appearance of a fast mode at low values 

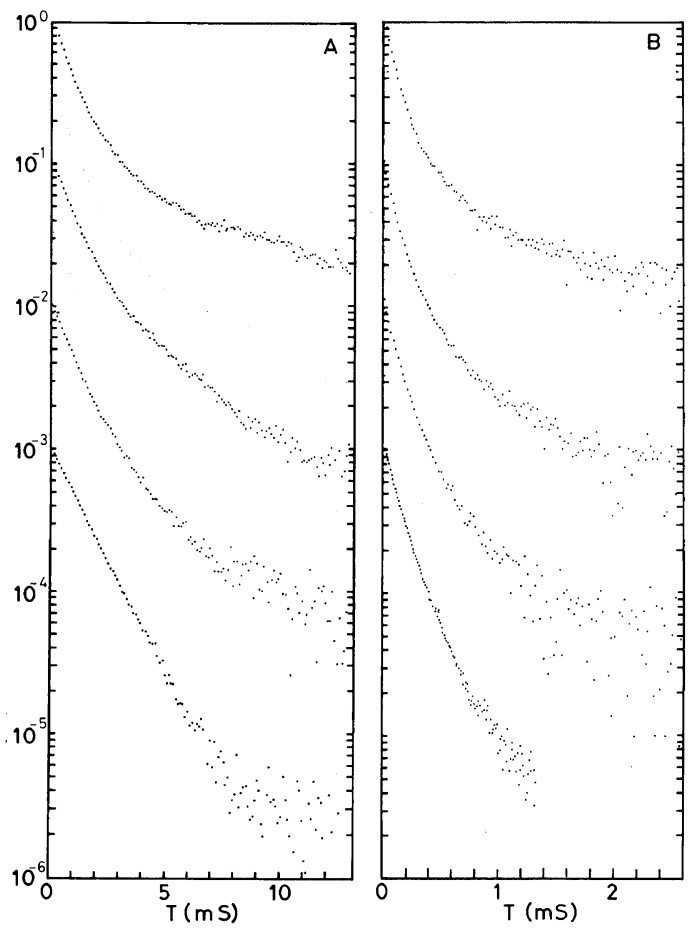

Figure 7. The effects of concentration on the correlation function for two values of $k_{\mathrm{s}}^{2}, 2.99 \times 10^{9} \mathrm{~cm}^{-2}$ (A) and $3.75 \times 10^{10} \mathrm{~cm}^{-2}(\mathrm{~B})$, are shown in this figure. In both plots, the concentration are, starting from the top, $6.25,5.6,4.4,3.6,3.0,2.5$ and $1.0 \mathrm{mg} \mathrm{cc}^{-1}$. For all concentrations, the initial values of the correlation function are chosen arbitarily.

of $k_{\mathrm{s}}^{2}$ where the effects of intramolecular interactions do not affect the correlation function. Figure 8 , a double exponential fit, shows this clearly. Figure 9 shows the relative amplitude, $A_{2} /\left(A_{1}+A_{2}\right)$, corresponding to the fast mode. The solid line in this figure shows the theoretical curve of $A_{2} /\left(A_{1}+A_{2}\right)$ calculated according to the free-draining model.

Figures 3 and 8 show that at $0.5 \mathrm{mg} \mathrm{cc}^{-1}$, the relation of $T_{2}^{-1}$ to $k_{\mathrm{s}}{ }^{2}$ is the same as for $0.1 \mathrm{mg} \mathrm{cc}^{-1}$. This implies $T_{2}$ is a relaxation time for internal motion. At $1.5 \mathrm{mg} \mathrm{cc}^{-1}$, a concentration very close to $c^{*}$, and for small values of $k_{\mathrm{s}}{ }^{2}, T_{2}^{-1}$ becomes small (Figure 8) and the amplitude which corresponds to $T_{2}$ becomes large (Figure 9). At $3.0 \mathrm{mg} \mathrm{cc}^{-1}$, twice $c^{*}$, $T_{2}$ is distinguishable from $T_{1}$ even for small values of $k_{\mathrm{s}}{ }^{2}$. Finally, at $5.0 \mathrm{mg} \mathrm{cc}^{-1}$, about three times $c^{*}, T_{2}^{-1}$ becomes linear in $k_{\mathrm{s}}{ }^{2}$. For the most concentrated sample, $10 \mathrm{mg} \mathrm{cc}^{-1}$, the double exponential analysis does not work well because of the multi-exponential

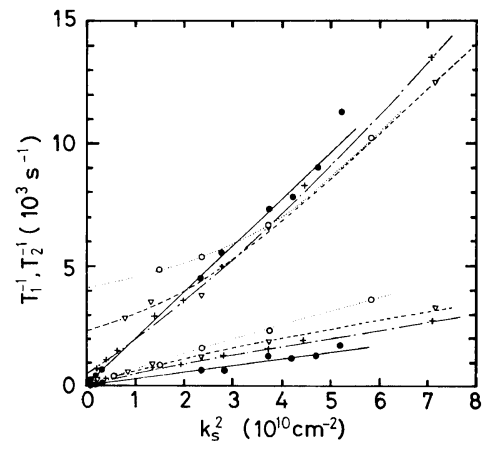

Figure 8. Results of the double exponential analysis for the semi-dilute region-I. The inverse relaxation times, $T_{1}^{-1}$ and $T_{2}{ }^{-1}$, are plotted against $k_{\mathrm{s}}{ }^{2}$. The concentrations are: $0.5 \mathrm{mg} \mathrm{cc}^{-1}(\bigcirc,---), 1.5 \mathrm{mg} \mathrm{cc}^{-1}(\nabla$, $---) \simeq c^{*}, 3 \mathrm{mg} \mathrm{cc}^{-1}\left(+,----_{--}\right)$, and $5 \mathrm{mg} \mathrm{cc}^{-1}$ - ).

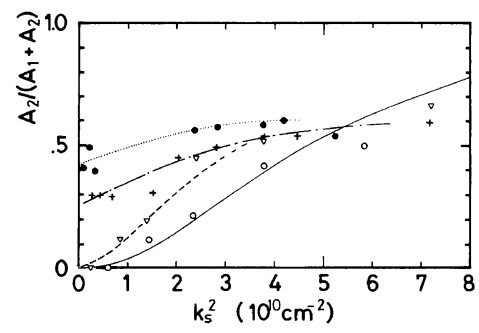

Figure 9. Results of the double exponential analysis for the semi-dilute region-II. The relative amplitudes, $A_{2} /\left(A_{1}+A_{2}\right)$, are plotted against $k_{\mathrm{s}}^{2}$. The solid line is the theoretical curve calculated using the free-draining model. The concentrations are: $0.5 \mathrm{mg} \mathrm{cc}^{-1}(\mathrm{O}), 1.5$ $\mathrm{mg} \mathrm{cc}^{-1}(\nabla,----), 3 \mathrm{mg} \mathrm{cc}^{-1}(+,----)$, and $5 \mathrm{mg} \mathrm{cc}^{-1}$ (ด, -----).

form of the correlation functions (see Figure 2). However, at this concentration, the inverse initial relaxation time, $\tau^{-1}$, satisfies the relation,

$$
\tau^{-1} \propto k_{\mathrm{s}}^{2}
$$

\section{DISCUSSION}

According to the bead and spring model of the chain polymer, the relaxation time of the lowest mode of internal motion, $\tau_{1}$, is given by,

$$
\tau_{1}=\beta b^{2} / 3 k T \lambda_{1}
$$

where $\beta$ is the friction coefficient of each segment, $b$ is the average segment length, and $\lambda_{1}$ is the smallest 
nonzero eigenvalue of the $N \times N$ matrix, $H A$, introduced by Zimm. ${ }^{7}$ When $N \gg 1$, which is true for macromolecules, we have in the free-draining case,

$$
\lambda_{1} \simeq \pi^{2} / N^{2}
$$

Using the free-draining relation,

$$
D=k T / \beta N
$$

and $\left\langle l_{\mathrm{e}}{ }^{2}\right\rangle=b^{2} N$ with eq 14 , we have,

$$
\tau_{1}=\left\langle l_{\mathrm{e}}^{2}\right\rangle / 3 \pi^{2} D
$$

The quantities, $D$ and $\left\langle l_{\mathrm{e}}{ }^{2}\right\rangle$ are macroscopic parameters of the molecule and can be measured using the QELS technique.

Substituting the measured values of $D$ and $\left\langle l_{\mathrm{e}}{ }^{2}\right\rangle$ into eq 17 , we obtain the value $\tau_{1}=0.476 \times 10^{-3} \mathrm{~s}$, which is very closed to the obtained value. However, it should be noted that high-molecular-weight polymers do not satisfy the free-draining relation, eq 16. Instead, polystyrene molecules in 2-butanone satisfy the well-known relation, $D \propto N^{-1 / 2}$ or $D \propto M_{w}^{-1 / 2}$ for $M_{w}$ in the range $10^{4} \sim 10^{7} .^{2}$ The observed value of $D=6.2 \times 10^{-8} \mathrm{~cm}^{2} \mathrm{~s}^{-1}$ is very close to $D=6.57 \times 10^{-8} \mathrm{~cm}^{2} \mathrm{~s}^{-1}$ calculated from the non-draining relation, ${ }^{11}$

$$
D=0.192 k T / \eta\left\langle l_{\mathrm{e}}{ }^{2}\right\rangle^{1 / 2}
$$

using $\left\langle l_{\mathrm{e}}^{2}\right\rangle^{1 / 2}=2980 \AA$. In the non-draining limit, Zimm et al. ${ }^{7}$ found,

$$
\lambda_{1}=16.6\left[\beta /\left(12 \pi^{3}\right)^{1 / 2} \eta b\right] N^{-3 / 2}
$$

where $\eta$ is the viscosity of the solvent. Substituting eq 19 into eq 14 we find,

$$
\tau_{1}=0.398\left\langle l_{\mathrm{e}}^{2}\right\rangle^{3 / 2} \eta / k T
$$

The quantity $D$ does not appear in this expression, and so, $\tau_{1}$ can be calculated using only $\left\langle l_{\mathrm{e}}^{2}\right\rangle$. For the case under consideration,

$$
\tau_{1}=1.038 \times 10^{-3} \mathrm{~s}
$$

This value is just twice our experimentally observed value.

Neither the value obtained for $\tau_{1}$, nor for the ratio $\tau_{1} / \tau_{2}$ can be explained by either the free-draining model or the non-draining model. The observed ratio of relaxation times of the first and second modes, $\tau_{1} / \tau_{2}$, is smaller than unity, while it is 4 and 3 in the free-draining and non-draining limits, respectively. ${ }^{12}$ The hydrodynamic interaction between segments reduces the friction for the long wavelength internal modes where adjacent segments move nearly in phase, but increases friction for short wavelength modes where adjacent segments move out of phase. ${ }^{13}$ This explains the difference between the values of $\tau_{1} / \tau_{2}$ in the non-draining and freedraining limits.

This, together with result for $\tau_{1}$, suggests that in the present condition, where there is no perturbation, such as an external flow of liquid, there is another interaction among the segments influencing the relaxation times in a manner similar to the hydrodynamic interaction.

As previously stated, the correlation function is observed to have the form of a double exponential curve for concentrations higher than $1 \mathrm{mg} \mathrm{cc}^{-1}$. At this concentration, the molecules begin to overlap. The slower relaxation time represents a diffusion of the whole polymer. The diffusion becomes slower as the concentration increases because of a monotonic increase in the viscosity of the solution. The plot of the inverse of either the faster or shorter relaxation time against $k_{\mathrm{s}}{ }^{2}$ changes dramatically as the concentration increases. At about $2 \sim 3$ times $c^{*}$, the concentration at which molecules start to overlap, the relation of $T_{2}$ to $k_{\mathrm{s}}{ }^{2}$ approaches $T_{2}^{-1} \propto k_{\mathrm{s}}{ }^{2}$, which is Fick's law.

This diffusion-like motion has its origin in an internal motion within a single polymer as shown in Figure 8 . When the polymer chains begin to overlap, the internal motion begins to involve more than a single chain. Eventually, at a concentration of about $5 \mathrm{mgcc}^{-1}$, all the molecules in the sample are involved in the internal motion. This appears in the $k_{\mathrm{s}}$ dependence of the change in shape of the correlation function. The change in shape of the curves first appears for large values of $k_{\mathrm{s}}$ and then spreads out to include the whole range of $k_{\mathrm{s}}$ values as the polymer concentration increases. At high concentrations, the internal motions include many touching polymers. This can be thought of as a diffusion in the number density of the segments.

The nature of this diffusion suggests that its relaxation time must have a strong relation to the internal motion of the individual polymers. This idea seems to be supported by the fact that at large values of $k_{\mathrm{s}}^{2}, T_{2}$ has the same value for a single polymer as for highly concentrated solutions.

Tanaka et al. ${ }^{14}$ demonstrated that in gels, the relaxation time of the number-density diffusion of segments fulfils Fick's law. Our experimental results 


\section{NisHIO and A. WADA}

fit this theory well. This diffusion of the number density of segments is also called cooperative diffusion. As this work shows, the cooperative diffusion has an origin different from the usual diffusion of polymers.

Acknowledgements. We wish to thank Prof. H. Nagasawa for kindly providing us with polystyrene molecules and Prof. T. Tanaka of M.I.T. for his helpful comments. We also should like to thank Dr. K. Soda and Dr. N. Suzuki of the University of Tokyo for their help on the data-analysis softwares and Mr. G. Swislow for his patient help with the English.

\section{REFERENCES}

1. R. Pecora, J. Chem. Phys., 49, 1032 (1968).

2. T. A. King, A. Knox, and J. D. G. McAdam, Chem. Phys. Lett., 19, 351 (1973).

3. Wu-Nan Huang and J. E. Frederick, Macromolecules,
7, 34 (1974).

4. E. Geissler and A. M. Hecht, J. Chem. Phys., 65, 103 (1976).

5. J. P. Munch, S. Candan, and G. Hild, J. Polym. Sci., $B, 15,11$ (1977).

6. J. D. G. McAdam and T. A. King, Chem. Phys., 6, 109 (1974).

7. B. H. Zimm, J. Chem. Phys., 24, 269 (1956).

8. P. E. Rouse, J. Chem. Phys., 21, 1272 (1953).

9. T. A. King, A. Knox, and J. D. G. McAdam, Polymer, 14, 293 (1973).

10. J. G. Kirkwood and J. Riseman, J. Chem. Phys., 16, 565 (1948).

11. H. Yamakawa, "Modern Theory of Polymer Solutions," Harper \& Row, Publishers, New York, N.Y., 1971, Chapter 6, p 272.

12. A. Perico, P. Piaggio, and C. Cuniberti, J. Chem. Phys., 62, 4911 (1975).

13. R. E. De Wames, W. F. Hall, and M. C. Shen, J. Chem. Phys., 46, 2782 (1967).

14. T. Tanaka, L. O. Hocker, and G. B. Benedek, J. Chem. Phys., 56, 5151 (1972). 